\title{
THE CHROMATIC POLYNOMIAL OF THE TRUNCATED ICOSAHEDRON ${ }^{1}$
}

D. W. HALL, J. W. SIRY, AND B. R. VANDERSLICE

The classical unsolved problem in the coloring of maps is to determine whether four colors are sufficient to color any map on a sphere. This problem has stimulated more general investigations such as the study of chromatic polynomials, ${ }^{2} P_{n}(\lambda)$, each of which gives the exact number of ways in which an associated map of $n$ regions can be colored using some or all of $\lambda$ different colors, [1]-[10]. It is sufficient, actually, to consider maps having triple vertices, for which $P_{n}(0)$ $=P_{n}(1)=P_{n}(2)=0$, hence more convenient expressions, such as

$$
Q_{n}(\lambda)=\frac{P_{n+3}(\lambda)}{\lambda(\lambda-1)(\lambda-2)},
$$

are often considered in practice. The study of chromatic polynomials has led to a conjecture of Birkhoff and Lewis which is a strong form of the four-color conjecture, [5]. They have shown that

$$
(\lambda-3)^{n} \ll Q_{n}(\lambda) \ll(\lambda-2)^{n}, \text { for } \lambda \geqq 5
$$

holds for all maps of triple vertices, and also that

$$
(\lambda-3)^{n} \ll Q_{n}(\lambda) \ll(\lambda-2)^{n}, \text { for } \lambda \geqq 4,
$$

holds for all such maps for which $0 \leqq n \leqq 8$. Here the relation between two polynomials, $f(x)$ and $g(x)$, which is symbolized by

$$
f(x) \ll g(x),
$$

holds if and only if the coefficients of $f(x)$ are non-negative and not greater than the corresponding coefficients of $g(x)$, and the relation between two polynomials, $S(y)$ and $T(y)$, which is symbolized by

Presented to the Society, March 14, 1953 under the title Chromatic polynomials of large maps and January 20,1959 under the title Chromatic polynomials; received by the editors July 11, 1963 and, in revised form, April 10, 1964.

${ }^{1}$ The completion of this work was made possible by a generous grant from the Research Foundation of the State University of New York.

${ }^{2}$ See [5] for fundamental principles and definitions underlying the treatment of chromatic polynomials. Numbers in brackets refer to the bibliography at the end of the paper. 


$$
S(y) \ll T(y), \text { for } y \geqq c,
$$

where $c$ is a constant, holds if upon setting $x=y-c, f(x)=S(y)$, and $g(x)=T(y)$, the relation (4) holds. Attempts to prove that (3) always holds for maps of triple vertices have broken down because of the possibility of the occurrence of maps which are regular in the sense of Birkhoff, [1]. By calculating the chromatic polynomials involved, Birkhoff and Lewis have shown that (3) does hold for what are probably the only maps of fewer than seventeen regions which are regular in this sense, and for one such map which has seventeen regions. The results of this calculation, [5], formed the basis for their conjecture to the effect that (3) always holds for maps of triple vertices. Their conjecture is actually a very strong form of the four-color conjecture, for if it were true, then, in particular, it would follow from (3) that $P_{n+3}(4) \geqq 24$. Hence the map $P_{n+3}$, associated with the chromatic polynomial $P_{n+3}(\lambda)$, could be colored in four colors.

The determination of the chromatic polynomials of large maps thus constitutes a valuable method for extending known results and testing conjectures. It is of particular interest to determine whether the conjecture of Birkhoff and Lewis holds in the case of the truncated icosahedron, which is the simplest regular map possessing the additional topological property that no two of its pentagons are contiguous. It was for this reason that the authors, in consultation with D. C. Lewis, felt that it would be of interest to determine whether or not the chromatic polynomial of the truncated icosahedron satisfies the conjecture mentioned above. It turns out that it does satisfy this conjecture.

The truncated icosahedron is obtained from a regular icosahedron by truncating the icosahedron at each of its twelve vertices. The map associated with the original icosahedron had twenty regions; the truncation process yields an additional twelve, so that the map associated with the truncated icosahedron has a total of thirty-two regions of which twelve are pentagons and twenty hexagons. When we speak of the "chromatic polynomial of the truncated icosahedron" we shall mean, of course, the chromatic polynomial of the map associated with the truncated icosahedron.

If a regular map is to be colored using some or all of $\lambda$ different colors then, to reduce the size of the coefficients, the chromatic polynomial is usually written in powers of $u=\lambda-3$. It is found that most maps cannot be colored in 0 or 1 or 2 or 3 colors, so that, for maps with this property, $u(u+1)(u+2)(u+3)$ is a factor of the chromatic polynomial. When a chromatic polynomial, $P_{n}(\lambda)$, is divided by this 
factor, the resulting quotient polynomial will be denoted by $Q_{n}(u)$. The chromatic polynomial to be presented in this paper is given in the latter form. Inasmuch as the chromatic polynomial of a map with $n$ regions is a polynomial of degree $n$, the chromatic polynomial which we obtain for the truncated icosahedron should be (after the division just indicated) of degree twenty-eight. The map for the truncated icosahedron is shown in Figure 1. The first term and the

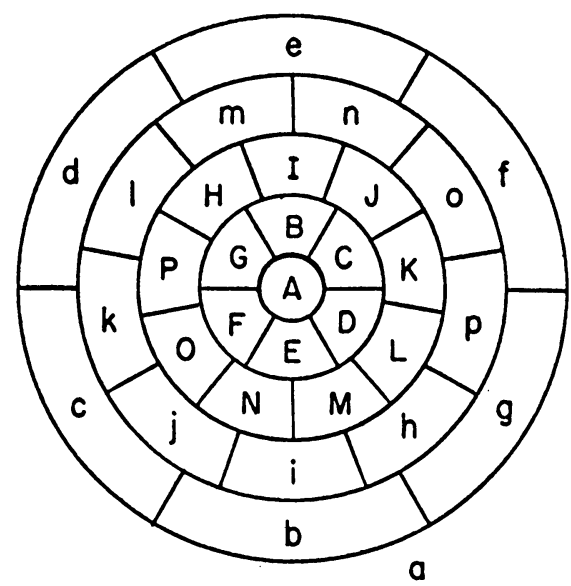

FIGURE 1

coefficients for the remaining terms of the chromatic polynomial for this map are given as follows:

$$
\begin{aligned}
u^{28} & +0+28-54+469-1,582+7,192-26,512 \\
& +97,763-333,760+1,076,100-3,222,960+8,888,435 \\
& -22,264,058+49,933,474-98,731,205+169,324,174 \\
& -247,428,839+301,855,436-299,843,126+234,327,105 \\
& -136,166,711+51,805,180-6,862,115-5,159,939 \\
& +3,702,618-1,143,882+181,952-11,994 .
\end{aligned}
$$

This paper contains an extremely condensed account of the way in which the above polynomial was obtained. In his dissertation (University of Maryland, 1953) J. W. Siry worked out a formula having as one of its special cases a result by means of which the chromatic polynomial of the truncated icosahedron could be computed if the chromatic polynomials for thirty-five other maps, each with twentysix or fewer regions, were known. 
Hall and Vanderslice have each independently checked the validity of the formula of Siry. Vanderslice, in her thesis (University of Maryland, 1948), described, and found the chromatic polynomials for, 155 maps not given by Birkhoff and Lewis. She had hoped to compute the chromatic polynomial for the truncated icosahedron, but this proved to be beyond computation with a desk computer until the discovery of the Siry formula. All 155 of the polynomials in the Vanderslice thesis have been computed independently and checked by Hall.

At this stage of the game, Hall and Vanderslice started a joint program to compute the chromatic polynomials for the thirty-five maps described by Siry, and hence the chromatic polynomial for the truncated icosahedron itself. Working independently, they completed the program, checking all polynomials. The set of thirty-five maps referred to above will be denoted by $T(26)$ and the related general set corresponding to the more general formula will be denoted by $S(n-6)$. Reference 8 contains a more detailed description of the relationships existing between the sets $S(n-6)$ and $T(26)$, as well as a definition of symmetric equivalence, a special form of topological equivalence which is of value in this context.

These sets and the formulas themselves were found by means of a method which can be sketched as follows. The discussion will be facilitated considerably if certain conventions are adopted in connection with the following reduction formulas of the type given by Birkhoff and Lewis:

$$
\begin{aligned}
& Q_{n}(u)=Q_{n}^{*}(u)-Q_{n-1}(u)+Q_{n-1}^{*}(u), \\
& Q_{n}(u)=(u+1) Q_{n-1}(u), \\
& Q_{n}(u)=u Q_{n-1}(u), \\
& Q_{n}(u)=(u-1) Q_{n-1}(u)+u Q_{n-2}(u)+Q_{n-2}^{*}(u),
\end{aligned}
$$

and

$$
\begin{aligned}
Q_{n}(u)= & (u-2) Q_{n-1}^{1}(u)+2(u-1) Q_{n-2}^{6}(u) \\
& +Q_{n-2}^{1}(u)+2 Q_{n-2}^{4}(u) .
\end{aligned}
$$

The latter is a simplified version of their pentagon reduction formula applicable to any map having a homeomorph which is symmetric about an axis of symmetry of the pentagon. The left member of a reduction formula will be referred to as the antecedent, and each term of 
the right member will be referred to as a consequent. In certain symbols used in the following discussion, the consequents of formulas (7), (10) and (11) will be denoted, respectively, by the digits $1,2,3, \cdots$, 8,9 , and 0 , and the consequents of formulas (8) and (9) will be denoted by a superscript bar and a prime, respectively. Regions of maps will be denoted by letters, and a side of two regions will be denoted by the letters associated with these two regions. In the case of each one of the formulas (7) through (11), the first consequent having the subscript $n-1$ denotes a map obtained from the antecedent by erasing a side which will be called the principal side. All the important information associated with the application of an entire formula such as the fundamental principle, (7), to a map such as the truncated icosahedron of Figure 1 can be given by means of a symbol of the type

$A C 123$

where the first two letters denote the principal side and the digits following serve to denote which formula was applied, and the resulting consequent maps as well. In the case of formulas (10) and (11) the first letter will serve also to denote the region being reduced. When sides are erased to obtain the maps denoted by the symbol 2 or 3 , the region label nearest to the beginning of the alphabet is assigned to the new region. In formulas (8) through (11), where one side of a region is erased, the label of the region being reduced is eliminated. When two sides are erased, the label of the region being reduced is retained. It is not necessary to specify the principal side in the case of the formulas (8) and (9), since these reductions possess symmetry. For simplicity, these reductions can be specified by adding the superscript bar or prime at the appropriate digit and giving the letter of the region being reduced following this symbol. For example, when the formula (12) is applied to the truncated icosahedron shown in Figure 1, the resulting map denoted by the symbol 3 contains a 3-gon labelled $C$. We erase one side of $C$ to obtain a map with one fewer regions, using (9). A prime is placed after the 3 , and a $C$ after the $3^{\prime}$ thus obtained so that our symbol (12) becomes

$$
A C 123^{\prime} C \text {. }
$$

This symbol conveys a great deal of information. It tells us that we broke down the truncated icosahedron by applying the fundamental principle (7) (this is the meaning of the $123^{\prime}$ in the formula) to obtain three new maps, denoted by 1,2 , and 3 . The map denoted by 3 contained a 3-gon labelled $C$, and we erased one side of this 3-gon to obtain the map $3^{\prime}$. Further reductions of the three maps $1,2,3^{\prime}$ are 
then completely specified by means of the symbols

$$
\begin{aligned}
& 1 A G 123^{\prime \prime} G A, \\
& 2 A G 123^{\prime} G, \\
& 3^{\prime} A G 1^{\prime} A 2 \overline{3}^{\prime \prime} A E G .
\end{aligned}
$$

Now (14) tells us how to break down the map 1 into three simpler maps $11,12,13^{\prime \prime}$, where $13^{\prime \prime}$ was obtained by erasing one side of each of the 3-gons $G$ and $A$. We have listed the breakdowns of all the maps we use in Table I, where each reduction is stopped when we reach a map in our set $T(26)$.

There are many times, during this reduction of the numerous maps under consideration, when we reach maps that have been encountered in our other reductions. To prevent duplication of effort, these maps are listed along with our symbols and an asterisk to warn the reader what has happened. When a map occurs in our reduction that is in the set $T(26)$, we show its symbol in bold face type to indicate that no further reduction is necessary.

After obtaining all 35 of the maps in $T(26)$, and computing and checking their chromatic polynomials, substitution in the special case of the formula of Siry given in (18) gives us the chromatic polynomial of the truncated icosahedron. This portion of the work was done by Hall and Vanderslice.

To illustrate the meanings of the symbols in Table I, we note that (16) is there written as

$$
3^{\prime} A G 1^{\prime *} A 2^{*} \overline{3}^{\prime \prime} \text { AEG }\left(13^{\prime \prime}, 23^{\prime}\right) \text {. }
$$

This tells us that when we reduce the map $3^{\prime}$ by applying (7) to the side $A G$, we obtain three maps $3^{\prime} 1,3^{\prime} 2,3^{\prime} 3$. We erase one side of the 3 -gon $A$ in $3^{\prime} 1$ to obtain $3^{\prime} 1^{\prime}$. The first asterisk in (17) comes after the $1^{\prime}$. This informs us that the map $3^{\prime} 1^{\prime}$ is chromatically equivalent to the map 13" (indicated in parentheses) which we reduce elsewhere in our computation. Similarly, $3^{\prime} 2$ is chromatically equivalent to $23^{\prime}$. Finally, the map $3^{\prime} 3$ is reduced by applying (8) and (9) to obtain $3^{\prime} \overline{3}^{\prime \prime}$, where the boldfacing serves notice that we have reached a map of the set $T(26)$.

Table I, which includes the symbols (13) through (16), contains the seven reduction sequences needed to reduce the truncated icosahedron to the maps of $T(26)$. The first of these seven sequences involves the central region of Figure 1. It yields six different configurations which are reduced in the remaining sequences. 


$$
\begin{aligned}
& P_{32}(\lambda)=\left(u^{6}-6 u^{5}\right. \\
& \left.+24 u^{4}-57 u^{3}+78 u^{2}-56 u+16\right) P_{26}(\lambda)\left(3^{\prime} \overline{3}^{\prime \prime}\right) \\
& +\left(u^{4}-3 u^{3}+6 u^{2}-10 u+6\right) P_{26}(\lambda)\left(13^{\prime \prime} 46\right) \\
& +\left(2 u^{5}-7 u^{4}+3 u^{3}+34 u^{2}-62 u+30\right) P_{26}(\lambda)\left(13^{\prime \prime} 64\right) \\
& +\left(2 u^{5}-12 u^{4}+36 u^{3}-44 u^{2}+18 u\right) P_{26}(\lambda)\left(228^{\prime} 4\right) \\
& +(6 u) P_{26}(\lambda)(2205)+\left(6 u^{2}-30 u+30\right) P_{26}(\lambda)(22042) \\
& +\left(u^{4}-12 u^{2}+32 u-18\right) P_{26}(\lambda)(22043) \\
& +\left(-6 u^{2}\right) P_{26}(\lambda)\left(11485^{\prime}\right) \\
& +\left(u^{4}-u^{3}-4 u^{2}+6 u\right) P_{26}(\lambda)\left(11405^{\prime}\right) \\
& +\left(3 u^{2}-u\right) P_{26}(\lambda)\left(23^{\prime} 25\right) \\
& +\left(-u^{4}+6 u^{3}-15 u^{2}+19 u-9\right) P_{26}(\lambda)\left(23^{\prime} 243\right) \\
& +\left(u^{5}-6 u^{4}+21 u^{3}-30 u^{2}+14 u\right) P_{26}(\lambda)(122345) \\
& +\left(5 u^{5}-18 u^{4}+21 u^{3}-5 u^{2}-3 u\right) P_{25}(\lambda)\left(13^{\prime \prime} 45^{\prime}\right) \\
& +\left(3 u^{3}-9 u^{2}+9 u-3\right) P_{25}(\lambda)\left(13^{\prime \prime} 56\right) \\
& +\left(3 u^{3}-6 u^{2}+3 u\right) P_{25}(\lambda)\left(13^{\prime \prime} 65\right) \\
& +\left(u^{6}-5 u^{5}+18 u^{4}-38 u^{3}+40 u^{2}-16 u\right) P_{25}(\lambda)\left(13^{\prime \prime} 445\right) \\
& \text { (18) }+\left(6 u^{3}-6 u^{2}\right) P_{25}(\lambda)\left(228^{\prime} 5\right)+(6 u) P_{25}(\lambda)\left(2206^{\prime}\right) \\
& +\left(2 u^{5}-6 u^{4}+28 u^{2}-24 u\right) P_{25}(\lambda)(220415) \\
& +\left(-6 u^{3}+6 u^{2}\right) P_{25}(\lambda)\left(114845^{\prime}\right) \\
& +\left(-6 u^{2}+6 u\right) P_{25}(\lambda)\left(114846^{\prime}\right) \\
& +\left(-6 u^{2}+6 u\right) P_{25}(\lambda)\left(114864^{\prime}\right)+(-6) P_{25}(\lambda)(114866) \\
& +(2 u-6) P_{25}(\lambda)(114066)+(3 u) P_{25}(\lambda)\left(23^{\prime} 26^{\prime}\right) \\
& +\left(u^{5}-6 u^{4}+18 u^{3}-23 u^{2}+10 u\right) P_{25}(\lambda)\left(122346^{\prime}\right) \\
& +\left(3 u^{3}-4 u^{2}\right) P_{25}(\lambda)(122355) \\
& +\left(u^{5}-4 u^{4}+6 u^{3}\right) P_{25}(\lambda)\left(1151155^{\prime}\right) \\
& +\left(u^{6}-5 u^{5}+18 u^{4}-38 u^{3}+40 u^{2}-16 u\right) P_{24}(\lambda)\left(13^{\prime \prime} 446^{\prime}\right) \\
& +\left(6 u^{3}-6 u^{2}\right) P_{24}(\lambda)\left(228^{\prime} 6^{\prime}\right) \\
& +\left(2 u^{5}-6 u^{4}+28 u^{2}-24 u\right) P_{24}(\lambda)\left(220416^{\prime}\right) \\
& +\left(2 u^{3}-6 u^{2}\right) P_{24}(\lambda)\left(114065^{\prime}\right)+\left(3 u^{3}-4 u^{2}\right) P_{24}(\lambda)\left(122356^{\prime}\right) \\
& +\left(3 u^{6}-9 u^{5}+3 u^{4}+9 u^{3}-6 u^{2}\right) P_{23}(\lambda)\left(13^{\prime \prime} 5 \overline{5}^{\prime}\right) \\
& +\left(-6 u^{3}-6 u^{2}\right) P_{23}(\lambda)\left(11486 \overline{5}^{\prime}\right) \text {. }
\end{aligned}
$$


TABLE I

Reduction Sequences

1. The truncated icosahedron $A C 123^{\prime} C$

$1 A G 123^{\prime \prime} G A$

$2 A G 1^{*} 23^{\prime} G(12)$

$3^{\prime} A G 1^{\prime *} A 2^{*} \overline{3}^{\prime \prime} A E G\left(13^{\prime \prime}, 23^{\prime}\right)$

$11 A E 456^{\prime *} E\left(13^{\prime \prime}\right)$

$12 A E 1^{*} 23^{\prime *} E\left(114,13^{\prime \prime}\right)$

$23^{\prime} A E 1^{*} 23^{\prime \prime *} A E\left(13^{\prime \prime}, 3^{\prime} 3^{\prime \prime}\right)$

$122 B D 1^{*} 2^{\prime *} C 3\left(22,23^{\prime} 2\right)$

2. The map $13^{\prime \prime}$

$C B 456$

$4 E B 45^{\prime} D 6$

$5 E C 4^{\prime *} D \overline{5} D L 6\left(13^{\prime \prime} 45^{\prime}\right)$

$6 E C 456^{*}\left(13^{\prime \prime} 56\right)$

$44 D B 4^{*} 56^{\prime} L\left(3^{\prime} 3^{\prime \prime}\right)$

3. The map 22

$E A 7^{*} 8^{\prime} D 9^{*} 0\left(1223,23{ }^{\prime} 2\right)$

$8^{\prime} B E 456^{\prime} I$

$0 B A 456^{\prime} I$

$04 A K 123$

$041 F A 4^{*} 56^{\prime} O\left(13^{\prime \prime} 64\right)$

4. The map $23^{\prime} 2$

$D A 456^{\prime} L$

$4 A J 12 * 3(13$ " 64$)$

$41 A N 1^{*} 2^{*} 3^{\prime *} A\left(13^{\prime \prime} 44,13^{\prime \prime} 46,13^{\prime \prime} 45^{\prime}\right)$
5. The map 114

$E B 7^{*} 89^{*} 0\left(115,13^{\prime \prime}\right)$

$8 G E 45^{\prime} F 6$

$0 G B \quad 45^{\prime} F 6$

$84 F E 4^{*} 5^{\prime} N 6^{\prime} O\left(13^{\prime \prime} 5\right)$

$86 C E 4^{\prime} D \overline{5}^{\prime} D L 6$

$04 C B 4^{*} 5^{*} 6^{*}(2204,22042,22043)$

$06 C E 4^{*} 5^{\prime} \mathrm{B} 6$ (22042)

6. The map 1223

$B A 456^{\prime *} I\left(23^{\prime} 25\right)$

$4 F A 4^{*} 56^{\prime} O\left(13^{\prime \prime} 44\right)$

$5 D B 4^{*} 56^{\prime} L(122345)$

7. The map 115

$A M 12 * 3 *(1148,1140)$

$1 A N 12 * 3 *\left(1148,13^{\prime \prime}\right)$

$11 C D 456$

$114 A F 12^{\prime *} G 3^{*}\left(23^{\prime} 24,12234\right)$

$115 G F 4^{*} 5^{\prime} A 6^{*}(22041,22042)$

$116 D N 1^{*} 2 * 3^{*} D(11404,11406$,

$\left.11405^{\prime}\right)$

$1141 G D 7^{*} 8^{\prime *} F 9^{*} 0^{*}\left(12234,228^{\prime} 4\right.$, $\left.23^{\prime} 24,2204\right)$

The chromatic polynomial of the truncated icosahedron (6) has twenty-eight zeros of which exactly four are real. We close this paper by listing approximations to all of these zeros. ${ }^{3}$

Approximations to the four real zeros are:

$$
\begin{array}{lr}
0.24697919, & 0.41539930, \\
0.52004593, & -0.38196601 .
\end{array}
$$

Approximations to the real and imaginary parts of the twelve pairs of conjugate complex zeros are given as follows:

3 These approximations were found on a high speed computer which was very generously made available to one of the authors by the Glendale Laboratories, Endicott, New York, of the International Business Machines Corporation. 


$\begin{array}{cc}\text { Real Parts } & \text { Imaginary Parts } \\ 0.63596985 & 0.030695947 \\ 0.70105547 & 0.30191011 \\ 0.88769325 & 0.48067209 \\ 0.54666090 & 1.9521084 \\ 0.83394414 & 1.0924455 \\ 0.86771617 & 0.77432779 \\ -0.96829388 & 3.0407459 \\ -0.20846283 & 2.7749345 \\ 0.82430230 & 1.5035971 \\ -1.8968529 & 3.0224596 \\ -2.8830096 & 2.3379808 \\ 0.25904794 & 2.3786634\end{array}$

\section{BIBLIOGRAPHY}

1. George D. Birkhoff, The reducibility of maps, Amer. J. Math. 35 (1912), 115128.

2. - A determinant formula for the number of ways of coloring a map, Ann. of Math. (2) 14 (1912), 42-46.

3. - On the number of ways of coloring a map, Proc. Edinburgh Math. Soc. (2) 2 (1930), 83-91.

4. - On the polynomial expressions for the number of ways of coloring a map, Ann. Scuola Norm. Sup. Pisa. (2) 3 (1934), 85-103.

5. George D. Birkhoff and D. C. Lewis, Chromatic polynomials, Trans. Amer. Math. Soc. 60 (1946), 355-451.

6. D. W. Hall and D. C. Lewis, Coloring six-rings, Trans. Amer. Math. Soc. 64 (1948), 184-191.

7. B. I. Rudebock, Chromatic polynomials, Thesis, University of Maryland, 1948.

8. J. W. Siry, Chromatic polynomials of large maps, Thesis, University of Maryland, 1953.

9. Hassler Whitney, A logical expansion in mathematics, Bull. Amer. Math. Soc. 38 (1932), 572-579.

10. - The coloring of graphs, Ann. of Math. (2) 33 (1932), 688-718.

Harpur College, State University of New York, Goddard Space Flight Center, National Aeronautics and Space Administration, and

UNIVERSITY OF MARYLAND 